\title{
A PROOF OF A THEOREM OF LUTTINGER AND SIMPSON ABOUT THE NUMBER OF VANISHING CIRCLES OF A NEAR-SYMPLECTIC FORM ON A 4-DIMENSIONAL MANIFOLD
}

\author{
Clifford Henry Taubes
}

\begin{abstract}
A proof is given of a theorem announced some years ago by Luttinger and Simpson to the effect that a compact 4-manifold that has a near-symplectic form in a given cohomology class admits one in the same class whose zero locus consists of any given, but strictly positive number of disjoint, embedded circles.
\end{abstract}

\section{Introduction}

A symplectic form on a smooth, oriented 4-manifold is a closed, 2-form whose square is nowhere vanishing and positive. A near symplectic form is a non-trivial, closed form whose square is non-negative and, on its zero locus, has rank 3 derivative. A number of years ago, Karl Luttinger and Carlos Simpson announced the following theorem:

Theorem 1. Let $n$ denote a positive integer. A smooth, oriented compact, connected 4-manifold that admits a near symplectic form in a given cohomology class admits one in the same class whose zero locus consists of the disjoint union of $n$ embedded circles.

No proof has been published. Having been asked at times about this theorem, and having quoted it on various occasions (see, e.g., [T1]), the author set about the task of providing a proof. This paper contains the author's proof of this theorem of Luttinger and Simpson. After writing this paper, the author learned that Tim Perutz $[\mathrm{P}]$ has recently proved the Luttinger-Simpson theorem along somewhat different lines.

To give some context to this theorem, note first that Hodge theory can be used to construct a non-trivial 2-form with non-negative square on any 4-manifold with postive self-dual, 2nd Betti number. Meanwhile, a folk theorem known to gauge theory afficianados from the work of Simon Donaldson in the 1980's (see [DK]) asserted that such a 2-form can be found with rank 3 derivative on its zero locus. Thus, its zero locus consists of some number of disjoint, embedded circles. A proof of this folk theorem was published by Honda [Ho].

By definition, a near symplectic form with no vanishing locus is symplectic. However, there are compact 4-manifolds with near symplectic forms but no symplectic ones [T2], [T3], [K]. Thus, any near symplectic form on such a manifold must have at least one component circle to its zero locus.

Received by the editors November 14, 2005.

Supported in part by the National Science Foundation. 
Theorem 1 has an analog for non-compact, but asymptotically Euclidean 4-manifolds. In this regard, a manifold is said to be asymptotically Euclidean when the complement of a compact set is diffeomorphic to the complement in $\mathbb{R}^{4}$ of a ball. A closed 2-form on such a manifold is deemed asymptotically standard when such a diffeomorphism pulls it back as $d x^{1} \wedge d x^{2}+d x^{3} \wedge d x^{4}$. Hodge theory with arguments like those used in [Ho] can be used to prove that every asymptotically Euclidean 4-manifold has an asymptotically standard, exact near-symplectic form.

Theorem 2. Let $n$ be a positive integer. Every smooth, oriented, asymptotically Euclidean 4-manifold has an asymptotically standard, exact, near symplectic form whose zero locus consists of the disjoint union of $n$ embedded circles.

A celebrated theorem of Gromov $[G]$ asserts that $\mathbb{R}^{4}$ is the only asymptotically Euclidean 4-manifold with an asymptotically standard symplectic form.

By the way, recent works of Kirby and Gay $[\mathrm{KG}]$ for the compact manifold case and Scott [S] for the case of an asymptotically Euclidean manifold construct near symplectic forms whose zero loci are determined apriori from a Kirby calculus presentation of the manifold. Near symplectic forms are used in [ADK] to study the differential topology of 4-manifolds. Applications towards this same end are conjectured in [T1].

The remainder of this article contains the proofs of Theorems 1 and 2.

\section{The birth of circles}

The purpose of this section is to prove the following proposition:

Proposition 2.1. Let $n$ denote a non-negative integer and let $X$ denote a smooth oriented 4-manifold with a near symplectic form whose zero locus consists of $n$ disjoint, embedded circles. Then $X$ has a cohomologically equivalent near symplectic form whose zero locus consists of $n+1$ disjoint, embedded circles.

Proof of Proposition 2.1. A newborn circle is constructed here by changing the original 2-form on a compact set in a coordinate chart that is disjoint from all zeros of the original form. As a theorem of Moser $[\mathrm{M}]$ asserts that all symplectic forms are locally symplectomorphic, the construction of a new vanishing circle is needed only for the case when the original form is the standard symplectic form on a ball in $\mathbb{R}^{4}$. This standard form is denoted in what follows as $\omega$; it is the form on $\mathbb{C}^{2}=\mathbb{R}^{4}$ that is given with respect to complex coordinates $(z, w)$ by

$$
\omega=\frac{i}{2}(d z \wedge d \bar{z}+d w \wedge d \bar{w}) .
$$

The plan is to modify $\omega$ only near the origin so that the result, $\omega^{\prime}$, is a near symplectic form that vanishes on a single circle. The description of this modification is given in five parts. The first part describes $\omega^{\prime}$ in a small ball about the origin, and the subsequent parts describe $\omega^{\prime}$ at successively larger distances from the origin through a distance beyond which $\omega^{\prime}=\omega$

Part 1: Let $\varepsilon$ denote a real number with absolute value less than 1 and suppose that $R \gg 1$. Now introduce the form $\mu=\mu(\varepsilon, R)$ given by

$$
\mu=\frac{i}{2}\left(-\varepsilon+|z|^{2}-|w|^{2}\right)(d z \wedge d \bar{z}+d w \wedge d \bar{w})+\frac{i}{2}(z w-R \bar{w}) d \bar{z} d \bar{w}-\frac{i}{2}(\bar{z} \bar{w}-R w) d z d w
$$


This form is closed, and is such that $\mu \wedge \mu \geq 0$. When $\varepsilon>0$, the zero locus of $\mu$ consists of two circles, these being

$$
\begin{array}{ll}
\text { - } & Z_{\varepsilon}=\left\{(z, w):|z|^{2}=\varepsilon \text { and } w=0\right\} . \\
\text { - } & Z_{\mathbb{R}}=\left\{(z, w):|w|^{2}=R^{2}-\varepsilon \text { and } z=R \bar{w} / w\right\} .
\end{array}
$$

In the case $\varepsilon<0$, there is only one vanishing circle; this is the locus

$$
\left\{(z, w):|w|^{2}=R^{2}-\varepsilon \text { and } z=R \bar{w} / w\right\} .
$$

Thus, as long as $R^{2} \gg|\varepsilon|$, the 1-parameter family $\varepsilon \rightarrow \mu(\varepsilon, R)$ sees the birth of a vanishing circle as $\varepsilon$ crosses zero from negative values to positive values. Of course, reversing the motion of $\varepsilon$ sees the death of a vanishing circle.

Now, fix $\varepsilon>0$ but much less than $10^{-10}$, and then fix $R$ so that $R \varepsilon^{1 / 2} \gg 1$. Fix some large number, T. A lower bound for $T$ is $10^{12} R \varepsilon^{1 / 2}$. The form $\omega^{\prime}$ on the ball of radius $\delta=(2 \varepsilon)^{1 / 2}$ about the origin is given by

$$
\omega^{\prime}=T^{-1} \mu .
$$

Part 2: Let $r=\left(|z|^{2}+|w|^{2}\right)^{1 / 2}$. This part describes $\omega^{\prime}$ where $\delta \leq r \leq 4 \delta$. For this purpose, fix a smooth, non-decreasing function on $[0, \infty)$ that has value 0 on $[0,1]$ and value 1 on $[2, \infty)$. Let $\beta$ denote the chosen function. When $\kappa>0$ has been specified, then $\beta_{\kappa}$ denotes the function on $\mathbb{R}^{4}$ that maps $(z, w)$ to $\beta\left(r^{2} / \kappa^{2}\right)$. Thus, $\beta_{\kappa}$ is zero where $r \leq \kappa$ and is equal to 1 where $r \geq 2^{1 / 2} \kappa$.

The form $\omega$ is exact; in particular, $\omega=d \alpha$ where

$$
\alpha=\frac{i}{4}(z d \bar{z}-\bar{z} d z)+\frac{i}{4}(w d \bar{w}-\bar{w} d w) .
$$

With $\alpha$ understood, define $\omega^{\prime}$ where $\delta \leq r \leq 4 \delta$ to equal

$$
\omega^{\prime}=T^{-1}\left(\mu+d\left(\beta_{\delta} \alpha\right)\right) .
$$

The form depicted in (2.7) is closed. Moreover, its square can be written as the product of the Euclidean volume form and the function $T^{-2} \sigma_{0}$ where

$$
\sigma_{0}=2 \theta^{2}+2 \delta^{-2} r^{2} \theta \beta_{\delta}^{\prime}+2\left|\left(\delta^{-2} \beta_{\delta}^{\prime}+1\right) z w-R \bar{w}\right|^{2} .
$$

Here, $\theta=\beta_{\delta}+\left(-\varepsilon+|z|^{2}-|w|^{2}\right)$ and $\beta_{\delta}^{\prime}$ is shorthand for the function $\left(\frac{d}{d t} \beta\right)\left(r^{2} / \delta^{2}\right)$. As explained momentarily, (2.8) depicts a positive function where $\delta \leq r \leq 4 \delta$.

To see that $\sigma_{0}>0$ where $\delta \leq r \leq 4 \delta$, note first that $\sigma_{0}$ is positive if $\theta$ is positive, and $\theta$ is positive unless $|w|^{2}+\varepsilon>|z|^{2}$. Since $\varepsilon=\frac{1}{2} \delta^{2}$ and $|w|^{2}+|z|^{2} \geq \delta^{2}$, this implies that $\theta$ is negative only where $|w|^{2}=\frac{1}{4} \delta^{2}$. This understood, $\theta \geq-3|w|^{2}$ in any event. Now suppose that the right most term in (2.8) is greater than $\frac{1}{2} R^{2}|w|^{2}$. When such is the case, the previous lower bound for $\theta$ implies that the expression in (2.8) is no less than

$$
2 \theta^{2}-48|w|^{2}+\frac{1}{2} R^{2}|w|^{2}
$$

This last expression is positive granted that $R^{2}>96$. As $R \geq 10$, the preceding inequality holds.

Meanwhile, the right most term in (2.8) is greater than $\frac{1}{2} R^{2}|w|^{2}$ unless

$$
\left|\left(\delta^{-2} \beta_{\delta}^{\prime}+1\right)\right| z|-R| \leq \frac{1}{2} R .
$$


This then requires that $|z| \geq \frac{1}{2} R \delta^{2}$. Under the circumstances, this last condition does not hold on the domain of interest because $\frac{1}{2} R \delta^{2}=2^{-1 / 2}\left(R \varepsilon^{1 / 2}\right) \delta$. Indeed, this is much greater than $4 \delta$ given that $R \varepsilon^{1 / 2}$ is much greater than 1 .

Part 3: This part describes the form $\omega^{\prime}$ where $4 \delta \leq r \leq 8 \delta$. To this end, note that where $r \geq 2 \delta$, the form that is depicted in (2.7) can be written as $T^{-1} \omega_{1}$ where

$$
\begin{aligned}
\omega_{1}= & (1+\varepsilon) \omega-\frac{i}{2} R(\bar{w} d \bar{z} d \bar{w}-w d z d w) \\
& +\left[\left(|z|^{2}-|w|^{2}\right) \omega+\frac{i}{2} z w d \bar{z} d \bar{w}-\frac{i}{2} \bar{z} \bar{w} d z d w\right] .
\end{aligned}
$$

Note in particular that the form that is depicted in the brackets on the far right in (2.11) has norm where $4 \delta \leq r \leq 8 \delta$ that is bounded by $10^{3} \delta^{2}$. Moreover, it can be written as $d \tau$ where $|\tau|=10^{5} \delta^{3}$. As a consequence, the norm of $d\left[\left(1-\beta_{4 \delta}\right) \tau\right]$ is bounded by $10^{6} \delta^{2}$.

Granted these last points, define $\omega^{\prime}$ where $4 \delta \leq r \leq 8 \delta$ to be

$$
\omega^{\prime}=(1+\varepsilon) \omega-\frac{i}{2} R(\bar{w} d \bar{z} d \bar{w}-w d z d w)+d\left[\left(1-\beta_{4 \delta}\right) \tau\right] .
$$

Because the norm of the right most term in (2.12) is smaller than $10^{6} \delta^{2}$, the square of the form that is depicted in (2.12) is nowhere zero where $4 \delta \leq r \leq 8 \delta$ if $\delta<10^{-4}$.

Part 4: This part describes $\omega^{\prime}$ where $8 \delta \leq r \leq 16 \delta$. To this end, choose a nondecreasing function, $\chi$, on $[0, \infty)$ with value $T^{-1}(1+\varepsilon)$ on $[0,1]$ and value 1 on $[2, \infty)$. Let $\chi_{8 \delta}$ denote the function $\chi\left(r^{2} /(8 \delta)^{2}\right)$ on $\mathbb{R}^{4}$. Let $\alpha$ denote the 1 -form in (2.6). Noting that

$$
\omega^{\prime}=T^{-1}(1+\varepsilon) d \alpha-\frac{i}{2} T^{-1} R \bar{w} d \bar{z} d \bar{w}+\frac{i}{2} T^{-1} R w d z d w
$$

where $r \sim 8 \delta$, the definition of $\omega^{\prime}$ extends to where $8 \delta \leq r \leq 16 \delta$ as

$$
\omega^{\prime}=d\left(\chi_{8 \delta} \alpha\right)-\frac{i}{2} T^{-1} R \bar{w} d \bar{z} d \bar{w}+\frac{i}{2} T^{-1} R w d z d w
$$

The square of the form that is depicted in (2.14) is a nowhere zero multiple of the Euclidean volume form. Indeed, the square is obtained by multiplying the volume form by the function

$$
2 \chi_{8 \delta}^{2}+2(8 \delta)^{-2} r^{2} \chi_{8 \delta} \chi_{8 \delta}^{\prime}+2\left|(8 \delta)^{-2} z w \chi_{8 \delta}^{\prime}-T^{-1} R \bar{w}\right|^{2}
$$

and this function is positive because $\chi_{8 \delta}^{\prime}=\left(\frac{d}{d t} \chi\right)\left(r^{2} /(8 \delta)^{2}\right)$ is non-negative.

Part 5: This last part describes $\omega^{\prime}$ where $r \geq 16 \delta$. To this end, note that the 2 -form $-\frac{i}{2} \bar{w} d \bar{z} d \bar{w}+\frac{i}{2} w d z d w$ can be written as $d \nu$ with $\nu=-\frac{i}{2}(\bar{w} \bar{z} d \bar{w}-w z d w)$. This understood, extend the definition of $\omega^{\prime}$ to where $r \geq 16 \delta$ using

$$
\omega^{\prime}=\omega+T^{-1} \operatorname{Rd}\left[\left(1-\beta_{16 \delta}\right) \nu\right] .
$$

This form has everywhere positive square provided that $T^{-1} R \delta \ll 10^{-6}$. Thus, as long as $T \gg 10^{6} R \delta=2^{1 / 2} 10^{6} R \varepsilon^{1 / 2}$, the form depicted in (2.16) is symplectic where $r \geq 16 \delta$. By design, it is equal to $\omega$ where $r \geq 32 \delta$. 


\section{Melding component circles}

Suppose that $\omega$ is a near symplectic form on a given 4-manifold whose zero locus is a smooth, embedded, union of circles. Let $Z$ denote this zero locus. Suppose that $p \neq p^{\prime}$ are points in $Z$. The purpose of the subsequent discussion is to describe a second near symplectic form that is cohomologous to $\omega$ and whose zero locus, $Z^{\prime}$, is a disjoint union of embedded circles with the following property: There exists a ball, $B_{0}$, that contains $p$ and $p^{\prime}$ and is such that

- $Z \cap B_{0}$ is the disjoint union of two intervals, $I$ and $I^{\prime}$, with $p \in I$ and $p^{\prime} \in I^{\prime}$.

- $Z \cap\left(M-B_{0}\right)=Z^{\prime} \cap\left(M-B_{0}\right)$.

- $Z^{\prime} \cap B_{0}$ is the disjoint union of two arcs that connect $\partial I$ to $\partial I^{\prime}$.

The new form is denoted in what follows by $\omega^{\prime}$.

Theorems 1 and 2 follow directly given Proposition 2.1 and the existence of the form $\omega^{\prime}$ as just described.

There are six parts to the construction of $B_{0}$ and $\omega^{\prime}$.

Part 1: Here is the strategy: The ball $B_{0}$ contains a smaller, closed ball, $B_{1}$, that is chosen to have the following four properties: First, $p$ and $p^{\prime}$ are in $B_{1}$ and $Z \cap B_{1}$ is a pair of disjoint arcs, one containing $p$ and the other $p^{\prime}$. Second, the inclusion $\iota: \partial B_{1} \rightarrow X$ is transversal to $Z$ and so $\partial B_{1}$ intersects $Z$ at four points. Third, $\iota^{*} \omega$. can be written as $d \alpha$ and $\alpha \wedge d \alpha \geq 0$ with equality only at the four points in $Z \cap \partial B_{1}$. In particular, $\alpha$ is a contact form on the complement in $\partial B_{1}$ of $Z \cap \partial B_{1}$. Finally, the contact structure that $\alpha$ defines on the complement of $Z \cap \partial B_{1}$ is overtwisted.

With the preceding understood, a diffeomorphism is constructed from $B_{1}$ to itself with certain special properties. First, the diffeomorphism interchanges two of the four points that comprise $Z \cap \partial B_{1}$; it fixes one boundary of $I$ and one of $I^{\prime}$ while interchanging the other boundary component of $I$ with that of $I^{\prime}$. Second, the diffeomorphism pulls $\omega$ back as itself in a neighborhood of four small radius balls that are centered on the four points of $Z \cap \partial B_{1}$. Third, the diffeomorphism pulls $\alpha$ back as itself near these same four point. Let $\psi$ denote this diffeomorphism. With the preceding understood, the form $\psi^{*} \alpha$ agrees with $\alpha$ near $Z \cap \partial B_{1}$ and is a contact form on the complement in $\partial B_{1}$ of $Z \cap \partial B_{1}$. Note that $\psi^{*} \alpha$ is overtwisted since $\alpha$ is overtwisted. Finally, the 2-plane fields $\operatorname{kernel}(\alpha)$ and $\operatorname{kernel}\left(\psi^{*} \alpha\right)$ are homotopic as 2-plane fields with fix boundary values.

Theorems of Eliashberg and Gray are invoked next to find a diffeomorphism, $\phi$ : $\partial B_{1} \rightarrow \partial B_{1}$ that restricts as the identity on a neighbhood of $Z \cap \partial B_{1}$, and is such that $\phi^{*} \psi^{*} \alpha=g \alpha$ with $g>0$ a function on $\partial B_{1}$ that equals 1 near $Z \cap \partial B_{1}$. With $\phi$ in hand, define a new manifold, $X^{\prime}$, by surgery on $X$ :

$$
X^{\prime}=\left(X-B_{1}\right) \cup_{\phi} B_{1} .
$$

Thus, $B_{1}$ is removed and then glued back using the diffeomorphism $\phi$. A theorem of Hatcher [Ha] says that $\phi$ is homotopic to the identity map of $\partial B_{1}$ via a 1-parameter family of diffeomorphisms of $B_{1}$. As a result, $X$ is diffeomorphic to $X^{\prime}$. Here is another consequence: Use (3.1) to define a canonical embedding, $v: X-B_{1} \rightarrow X^{\prime}$. Let $B_{0} \subset X$ denote a ball that contains $B_{1}$ in its interior. There exists a diffeomorphism $\lambda: X \rightarrow X^{\prime}$ such that $\lambda=v$ on $X-B_{0}$. 
The final step argues that the form $\omega$ on $X-B_{1}$ can be modified in a small neighborhood of $B_{1}$ so as to match up smoothly across $\partial B_{1}$ with a small, positive, constant multiple of the form $\phi^{*} \psi^{*} \omega$. The two thus define a smooth, near-symplectic form on $X^{\prime}$ whose zero locus is a disjoint union of embedded circles. Take $\omega^{\prime}$ to be the pull back via the diffeomorphism $\lambda$ of this near symplectic form on $X^{\prime}$.

Part 2: This part of the story describes the ball $B_{1}$. This ball is obtained by smoothing a $C^{1}$ ball that is obtained as the union of five parts. Two of these parts consist of a pair that lie near $p$ and two consist of an analogous pair near $p^{\prime}$. The remaining part is a small radius, tubular neighborhood of a path between $p$ and $p^{\prime}$. The interior of this path should be disjoint from $Z$, and it is constrained near its endpoints. These five parts are described in turn.

To consider the parts near $p$, remark first that the form $\omega$ can be modified near its zero locus so that the modification has the same zero locus as the original, and such that any given point on the zero locus has a neighborhood with coordinates $(t, x, y, z)$ for which the modified form appears as:

$$
d t(x d x+y d y-2 z d z)+x d y d z-y d x d z-2 z d x d y .
$$

Thus, the zero locus is the $t$-axis in this coordinate chart. The Euclidean metric defines a metric for such a coordinate chart. Such modifications near the points $p$ and $p^{\prime}$ are assumed implicitly in what follows; the modified form is denoted by $\omega$ as was the original.

Suppose now that $(t, x, y, z)$ are coordinates as just described that are centered on the point $p$. Fix a small real number, $\varepsilon>0$, and let $C_{-}$denote the half ball in the coordinate system given by the conditions

$$
C_{-}=\left\{(t, x, y, z): t^{2}+x^{2}+y^{2}+z^{2}=\varepsilon^{2} \text { and } z \leq 0\right\} .
$$

This $C_{-}$is the first of the pair of components that defines the ball $B_{1}$.

The second component is denoted as $C_{+}$. The specification of $C_{+}$requires the choice of a small, positive number, $\delta>0$. I shall take $\delta \gg \varepsilon$, and this will require that $\varepsilon$ be very small. Here is $C_{+}$:

$$
C_{+}=\left\{(t, x, y, z): t^{2}+x^{2}+y^{2} \leq \varepsilon^{2} \text { and } 0 \leq z \leq \delta\right\} .
$$

Thus, $C_{-} \cup C_{+}$is a half-ball that extends a distance $\delta$ along the positive $z$ axis.

Analogous versions of $C_{-}$and $C_{+}$are defined near $p^{\prime}$ using the $p^{\prime}$ version of the coordinates $(t, x, y, z)$. These are denoted in what follows by $C_{-}^{\prime}$ and $C_{+}^{\prime}$.

To define the final component of $B_{1}$, choose an embedded arc, $\gamma:[-1,1] \rightarrow X$ such that $\gamma(-1)=p$ and $\gamma(1)=p^{\prime}$. Require that $\gamma$ have the following properties: First, its interior is disjoint from $Z$. Second $\gamma$ coincides where its affine parameter is near -1 with a segment of the positive $z$ axis. To be precise, assume that

$$
\gamma(s)=(t=0, x=0, y=0, z=s+1)
$$

for $-1 \leq s \leq-1+\delta$. An analogous constraint as defined using the $p^{\prime}$ version of the coordinates $(t, x, y, z)$ is required near $s=1$. In this case, $\gamma$ should coincide where its affine parameter is near 1 with a segment of the negative $z$ axis; thus $\gamma(s)=(0,0,0, s-1)$ when $1-\delta \leq s \leq 1$.

Granted (3.5), the fifth part of $B_{1}$ consists of the radius $\varepsilon$ tubular neighborhood of the portion of $\gamma$ where $-1+\delta \leq s \leq 1-\delta$. Use $C_{0}$ to denote this portion of $B_{1}$. 
Note that $\partial B_{1}$ is a $C^{1}$ submanifold, but not $C^{2}$. The failure of differentiablility occurs where $C_{+}$joins to $C_{-}$, thus on the 2-sphere where $z=0$ and $t^{2}+x^{2}+y^{2}=\varepsilon^{2}$. One way to rectify this is to replace $C_{-}$as follows: Fix some $\varepsilon_{1}>0$ but very small so that $\varepsilon_{1} \ll \varepsilon$. Let $f:[0,1] \rightarrow[0, \infty)$ denote a smooth, non-decreasing function with $f(t)=t$ where $t \geq \varepsilon_{1}^{2}$ and $f(t)=0$ where $t \leq \frac{1}{2} \varepsilon_{1}^{2}$. Now set

$$
C_{-}^{\prime}=\left\{(t, x, y, z): t^{2}+x^{2}+y^{2}+f\left(z^{2}\right) \leq \varepsilon^{2} \text { and } z \leq 0\right\} .
$$

Part 3: This part describes the 1-form $\alpha$. To this end, I first define $\alpha$ on the part of $\partial B_{1}$ in $C_{+}$. To do so, I write $t=r \cos (\lambda), x=r \sin (\lambda) \cos (\varphi)$ and $y=r \sin (\lambda) \sin (\varphi)$; I then observe that $\omega$ on $C_{+}$is given by

$$
\begin{aligned}
& \omega=r^{2} \sin (\lambda) d r d \lambda-2 z \cos (\lambda) d r d z+2 z r \sin (\lambda) d \lambda d z \\
& \quad+r^{2} \sin ^{2}(\lambda) d \varphi d z-2 z r \sin ^{2}(\lambda) d r d \varphi-2 z r^{2} \sin (\lambda) \cos (\lambda) d \lambda d \varphi .
\end{aligned}
$$

This understood, the pull-back of $\omega$ to the part of $\partial B_{1}$ in $C_{+}$is

$$
\omega_{+}=2 r z \sin (\lambda) d \lambda d z-r^{2} \sin ^{2}(\lambda) d z d \varphi-2 r^{2} z \sin (\lambda) \cos (\lambda) d \lambda d \varphi .
$$

Granted (3.8), note that $\omega=d \alpha_{+}$with

$$
\alpha_{+}=-r^{2} z \sin ^{2}(\lambda) d \varphi-2 r \cos (\lambda) z d z+\frac{1}{3} r^{3} \sin (\lambda) d \lambda .
$$

Thus, upon restriction to $\partial B_{1}$, one has $\omega_{+}=d \alpha_{+}$. In addition:

$$
\alpha_{+} \wedge d \alpha_{+}=\left[\frac{1}{3} r^{2} \sin ^{2}(\lambda)+2 z^{2}\left(1+\cos ^{2}(\lambda)\right)\right] r^{3} \sin (\lambda) d \lambda d \varphi d z
$$

This 3 -form vanishes only where $x=y=z=0$.

Consider next the story for $C_{-}^{\prime}$. On the portion of $\partial B_{1}$ in $C_{-}$near where $z>-\varepsilon$, the function $r$ becomes a function of $z$ via

$$
r=\left(\varepsilon^{2}-f\left(z^{2}\right)\right)^{1 / 2} .
$$

As a consequence, the pull-back of $\omega$ to the portion of $\partial B_{1} \cap C_{-}^{\prime}$ were $z>-\varepsilon$ is

$$
\begin{aligned}
\omega_{-}=\left(-r^{2} r_{z}+2 z r\right) \sin (\lambda) d \lambda d z-\left(r^{2}+2 r z r_{z}\right) \sin ^{2}(\lambda) d z d \varphi \\
-2 z r^{2} \sin (\lambda) \cos (\lambda) d \lambda d \varphi .
\end{aligned}
$$

Note that I can write $\omega_{-}$as $d \alpha_{-}$with

$$
\alpha_{-}=-r^{2} z \sin ^{2}(\lambda) d \varphi-2 r \cos (\lambda) z d z+\frac{1}{3} r^{3} \sin (\lambda) d \lambda .
$$

A calculation finds that

$$
\alpha_{-} \wedge d \alpha_{-}=\left[\frac{1}{3}\left(\left(r^{2}+z^{2} f^{\prime}\right) \sin ^{2}(\lambda)+2 z^{2}(1+\cos 2(\lambda))\right] r^{3} \sin (\lambda) d \lambda d \varphi d z\right.
$$

A change of coordinates near where $z=-\varepsilon$ finds that $\alpha_{-}$is smooth on this locus also. The 3-form that is depicted in (3.14) vanishes only on the locus where $z=x=y=0$.

A comparison between (3.13) and (3.9) finds that $\alpha_{-}$smoothly extends $\alpha_{+}$from the $C_{+}$portion of $\partial B_{1}$ to the $C_{-}$portion.

Here is one last observation: The contact structure just described in overtwisted. Indeed, the circle where $z=0$ and $\cos (\lambda)=0$ is tangent to the contact plane field, but bounds a disk in the portion of $\partial B_{1}$ in $C_{-} \cup C_{+}$that avoids the two points 
where the contact form vanishes and is transverse to the contact plane field along its boundary. This disk is obtained as a perturbation of the disk that is defined by the conditions $z=0$ and $\frac{1}{2} \pi \leq \lambda \leq \pi$; the pertubation has $z$ becoming slightly negative as $\lambda$ approaches $\pi$. The existence of a disk with these properties characterizes an overtwisted contact structure.

An analogous contact form should be defined on the part of $\partial B_{1}$ in $C_{-}^{\prime} \cup C_{+}^{\prime}$. The $C_{+}^{\prime}$ part of the latter is denoted below by $\alpha_{+}^{\prime}$.

The next task is to extend the contact structure just described to the portion of $\partial B_{1}$ that lies in $C_{0}$. To this end, observe that for $z \sim \delta$ on the part of $\partial B_{1}$ in $C_{+}$, the contact form $\alpha_{+}$can be written as

$$
-\tau d k-z b^{2} d \varphi+\frac{1}{3} r^{3} \sin (\lambda) d \lambda
$$

where $b=r \sin (\lambda), \tau=r \cos (\lambda)$ and $k=z^{2}-1$. Meanwhile, for $z^{\prime} \sim-\delta$ on the part of $\partial B_{1}$ in $C_{+}^{\prime}$, the contact form $\alpha_{+}^{\prime}$ can be written as

$$
-\tau^{\prime} d k^{\prime}-\left|z^{\prime}\right| b^{\prime 2} d\left(-\varphi^{\prime}\right)+\frac{1}{3} r^{3} \sin \left(\lambda^{\prime}\right) d \lambda^{\prime}
$$

where $b^{\prime}=r \sin \left(\lambda^{\prime}\right), \tau^{\prime}=r \cos \left(\pi-\lambda^{\prime}\right)$, and $k^{\prime}=1-z^{\prime 2}$. Note in this regard that the change of coordinates from $\left(z^{\prime}, \lambda^{\prime}, \varphi^{\prime}\right)$ to $\left(z^{\prime}, \pi-\lambda^{\prime},-\varphi^{\prime}\right)$ defines an oriented map that extends over $C_{+}^{\prime} \cup C_{-}^{\prime}$ as the map $\left(t^{\prime}, z^{\prime}, x^{\prime}, y^{\prime}\right) \rightarrow\left(-t^{\prime}, z^{\prime}, x^{\prime},-y^{\prime}\right)$.

Here is a crucial point: The function $k=z^{2}-1$ restricts to the $-1<s \leq-1+\delta$ part of $\gamma$ as an increasing function of $s$. It is also the case that $k^{\prime}=1-z^{\prime 2}$ restricts to the part of $\gamma$ where $1-\delta \leq s<1$ as an increasing function of $s$. As a consequence, there is an oriented diffeomorphism, $s \rightarrow \sigma(s)$, that sends $(-1,1)$ to $(-1,1)$ and is such that $k=\sigma$ where $\sigma \sim-1+\delta^{2}$, and such that $k^{\prime}=\sigma$ where $\sigma \sim 1-\delta^{2}$.

To proceed, it is worth considering what $\omega$ looks like on a neighborhood of an embedded path in $X$ that avoids $Z$. I'll denote this path by $\gamma$. Choose a coordinate system $\left(\sigma, v_{1}, v_{2}, v_{3}\right)$ on a tubular neighborhood of $\gamma$ so that $\gamma=\{(-, 0,0,0)\}$. Then

$$
\left.\omega\right|_{\gamma}=d \sigma \wedge A_{i}(\sigma) d v_{i}+\varepsilon_{i j k} B_{k}(\sigma) d v_{i} \wedge d v_{j},
$$

where $B_{i} A_{i}>0$ at all values of $\sigma$. I can change coordinates $w_{1}=A_{i}(\sigma) v_{i}$ so that

$$
\left.\omega\right|_{\gamma}=d \sigma \wedge d w_{1}+2 B_{1} d w_{2} \wedge d w_{3}+\left(B_{2} d w_{3}-B_{3} d w_{2}\right) \wedge d w_{1}
$$

after redefining the collection $\left\{B_{i}\right\}$. Note that $B_{1}>0$. Change coordinates again, this time to coordinates $\left(\sigma, c_{1}, c_{2}, c_{3}\right)$ with $\left(c_{1}, c_{2}, c_{3}\right)$ given by

$$
w_{1}=c_{1}, w_{2}=-m B_{1}^{-1 / 2}\left(c_{2}+\left(B_{2} / B_{1}\right) c_{1}\right), w_{3}=m B_{1}^{-1 / 2}\left(c_{3}+\left(B_{3} / B_{3}\right) c_{1}\right)
$$

here $\sigma \rightarrow m(\sigma)$ is a favorite, strictly positive function on the domain of $\sigma$. With respect to these new coordinates,

$$
\left.\omega\right|_{\gamma}=d \sigma \wedge d c_{1}-2 m(\sigma) d c_{2} \wedge d c_{3}
$$

Now consider extending $\omega$ off of $\gamma$. To this end, fix in advance a smooth function $\sigma \rightarrow n(\sigma)$ and then use Moser's procedure [M] to find a tubular neighborhood of $\gamma$ with coordinates $\left(\sigma, c_{1}, c_{2}, c_{3}\right)$ where $(3.20)$ can be extended as

$$
\begin{aligned}
\omega=d \sigma \wedge d c_{1}+m^{\prime}\left(c_{2} d c_{3}-c_{3} d c_{2}\right) & \wedge d \sigma \\
& -2 m d c_{2} \wedge d c_{3}+\frac{1}{2} d c_{1} \wedge d\left[n(\sigma)\left(c_{2}^{2}+c_{3}^{2}\right)\right] .
\end{aligned}
$$


These coordinates are unique up to a Hamiltonian diffeomorphism that preserves $\gamma$.

To say more, write $\omega$ as in (3.21) on the tubular neighborhood and note that writing $c_{1}=r \cos (\theta), c_{2}=r \sin (\theta) \sin (\phi)$ and $c_{3}=r \sin (\theta) \cos (\phi)$, finds $\omega=d a$ with

$$
a=-r \cos (\theta) d \sigma-m(\sigma) r^{2} \sin ^{2}(\theta) d \phi+\frac{1}{3} n(\sigma) r^{3} \sin (\theta) d \theta .
$$

This looks very much like what is written in (3.15) with the following identifications: First, $k=\sigma$ while $m(\sigma)=z$ and $n(\sigma)=1$ when $\sigma \sim-1+\delta^{2}$. Second, $\theta=\lambda$ and $\phi=\varphi$ when $\sigma \sim-1+\delta^{2}$. It also looks much like what appears in (3.16) with the identifications $k^{\prime}=\sigma, m(\sigma)=\left|z^{\prime}\right|, n(\sigma)=-1, \theta=-\lambda^{\prime}$ and $\phi=-\varphi^{\prime}$ when $\sigma \sim 1+\delta^{2}$.

These identifications can be made if $c_{1}=t$ near $p$ and $c_{1}=-t^{\prime}$ near $p^{\prime}$ and $\left(c_{2}, c_{3}\right)=$ $(x, y)$ near $p$; meanwhile $c_{1}=-t^{\prime}$ and $\left(c_{2}, c_{3}\right)=\left(-x^{\prime}, y^{\prime}\right)$ near $p^{\prime}$. This last set of identifications can be arranged with no difficulties when the tubular neighborhood has small radius (thus $\varepsilon$ is very small).

Note finally that the restriction of $\omega$ as depicted in (3.21) to the locus where $r=\left(c_{1}^{2}+c_{2}^{2}+c_{3}^{2}\right)^{1 / 2}=\varepsilon$ is the form

$$
\begin{aligned}
\omega_{0}=\left(1-\frac{1}{3} n^{\prime} r^{2}\right) r \sin (\theta) d \theta \wedge d \sigma-m^{\prime} r^{2} \sin ^{2}(\theta) d \sigma \wedge d \phi & \\
& -2 m r^{2} \sin (\theta) \cos (\theta) d \theta \wedge d \phi .
\end{aligned}
$$

In addition,

$$
a \wedge \omega_{0}=\left(2 m \cos ^{2}(\theta)+m\left(1-\frac{1}{3} n^{\prime} r^{2}\right) \sin ^{2}(\theta)+\frac{1}{3} n m^{\prime} r^{2} \sin ^{2}(\theta)\right) r^{3} \sin (\theta) d \sigma d \theta d \phi,
$$

which is positive provided that $r$ is small.

Thus, taking $\alpha=a$ on the part of $\partial B_{1}$ in $C_{0}$ extends the definition of $\alpha$ to the whole of $\partial B_{1}$ as a contact form for the restriction of $\omega$ to $B_{1}$.

Part 4: This part of the discussion concerns the diffeomorphism $\psi$. As noted at the very outset, each point on the zero locus of $\omega$ has a neighborhood with coordinates in which $\omega$ appears as depicted in (3.2). In particular, this is the case for the four points where the zero locus intersects $\partial B_{1}$. This understood, there is no obstruction to demanding that $\psi$, as it permutes these points, maps balls about the points so as to identify the version of $(t, x, y, z)$ in the domain ball with the corresponding version in the range ball. This will insure that $\psi^{*} \omega=\omega$ and $\psi^{*} \alpha=\alpha$ on these four balls.

It is proves convenient when discussing the 2 -plane fields $\operatorname{kernel}(\alpha)$ and $\operatorname{kernel}\left(\psi^{*} \alpha\right)$ to make a further constraint on $\psi$ beyond the requirement that it permute the four balls described above. To describe this additional constraint, agree to fix a small ball in $\partial B_{1}$ about each of the zeros of $\omega$ where the coordinates used in (3.2) are valid. Let $S \subset \partial B_{1}$ denote the complement of these four balls. This $S$ can be viewed as the complement inside the unit ball of $\mathbb{R}^{3}$ of three smaller open balls, $b_{0}, b_{1}$ and $b_{2}$. To be explicit, take these smaller balls to have radius $\frac{1}{100}$, with the center of $b_{0}$ at $\left(-\frac{1}{25},-\frac{1}{25}, 0\right)$, the center of $b_{1}$ at $\left(\frac{1}{25}, 0,0\right)$ and the center of $b_{2}$ at $\left(0, \frac{1}{25}, 0\right)$. The map $\psi$ will send $b_{1}$ to $b_{2}$ while fixing $b_{0}$ and the boundary of the 3 -ball. Let $L$ denote the 2-complex that is obtained from $\partial b_{0} \cup \partial b_{1} \cup \partial b_{2}$ by adjoining three arcs, these the respective shortest arcs between the origin and $\partial b_{0}, \partial b_{1}$ and $\partial b_{2}$. Let $L$ denote this 2 -complex. Here is a picture of $L$ : 


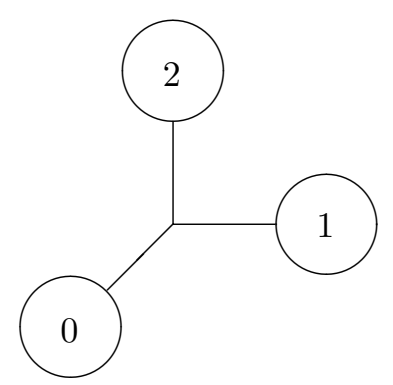

A sketch of $L$.

The map $\psi$ can be constructed so as to map $L$ to itself and so that $\psi^{*}(\alpha)=\alpha$ on a neighborhood of $b_{0} \cup b_{1} \cup b_{2}$ and on a neighborhood of the boundary of the unit ball in $\mathbb{R}^{3}$.

Keeping in mind that $\alpha$ and $\psi^{*} \alpha$ agree on the boundary of $S$, the final point here is made by

Lemma 3.1. The kernel of $\psi^{*} \alpha$ can be homotoped as a 2-plane field to the kernel of $\alpha$ with no change along the homotopy on the boundary of $S$.

Proof of Lemma 3.1. Frame $T^{*} S$ using the standard coordinate framing from $\mathbb{R}^{3}$. As an oriented 2-plane field on $S$ is the kernel of a nowhere zero 1-form on $S$, this fixed framing identifies any given 2-plane field with a map from $S$ to $S^{2}$, and it identifies any given map from $S$ to $S^{2}$ with a 2-plane field. This understood, the kernels of $\alpha$ and $\psi^{*} \alpha$ are homotopic as 2-plane fields (rel $\partial S$ ) if and only if the corresponding maps to $S^{2}$ are homotopic rel $\partial S$. To prove that such a homotopy exists, note that $S$ can be written as $\left(\left(S^{2} \times[0,1]\right) \cup L\right) / \sim$, where the equivalence relation identifies $S^{2} \times\{0\}$ with $L$ via a surjective map $f: S^{2} \rightarrow L$. This follows from the fact that $L$ has a regular neighborhood whose boundary is a 2 -sphere. This picture of $S$ identifies the boundary of the unit ball with $S^{2} \times\{1\} \subset S$.

Let $\sigma: S \rightarrow S^{2}$ denote a given map to $S^{2}$. Agree to identify the image $S^{2}$ with the unit sphere in the Lie algebra of $S U(2)$. The pull-back of $\sigma$ from $S$ to $S^{2} \times[0,1]$ can be lifted as a map to $S U(2)$ in the following manner: The lift, $h: S^{2} \times[0,1] \rightarrow S U(2)$ is such that $\sigma(z, t)=h(z, t) \sigma(z, 0) h^{-1}(z, t)$ and such that $h(z, 0)=\mathbb{I} \in S U(2)$. Note that $h$ is not unique; it can be modified by $h \rightarrow h \exp (u \sigma)$ where $u: S^{2} \times[0,1] \rightarrow$ $\mathbb{R} /(2 \pi \mathbb{Z})$.

If $\sigma$ and $\sigma^{\prime}$ are two maps from $S$ to $S^{2}$ that agree on $S^{2} \times\{1\}$, then the corresponding lifts $h$ and $h^{\prime}$ can be chosen so as to agree on $S^{2} \times\{1\}$ as well. This being the case, then the map from $S^{2} \times[0,1]$ to $S^{2}$ that sends $(z, t)$ to $h^{\prime}(z, 2 t)$ for $t \leq \frac{1}{2}$ and to $h(z, 2-2 t)$ for $t \geq \frac{1}{2}$ is a map from $S^{2} \times[0,1]$ to $S U(2)$ that restricts to both $S^{2} \times\{0\}$ and $S^{2} \times\{1\}$ as the identity. Let $\mu$ denote a volume form on $S U(2)$ with volume 1 . Then the integral over $S^{2} \times[0,1]$ of the pull-back of this form by $g$ is an integer, this denoted in what follows by $n_{h, h^{\prime}}$. It is relevant only by virtue of the following fact: Given maps $h$ and $h^{\prime}$ as just described are homotopic if and only if $n_{h, h^{\prime}}=0$. Note for reference below that this integer is zero in the case where $h$ is defined by $\alpha$ and $h^{\prime}$ by $\psi^{*} \alpha$; this is because $h^{\prime}$ can be taken to equal $\psi^{*} h$. This last conclusion requires that $\psi$ map $L$ to itself. 
The preceding observation does not imply that the respective maps from $S$ to $S^{2}$ defined by a and $\psi^{*} \alpha$ are homotopic. One further item is needed. To explain, let $\sigma: S \rightarrow S^{2}$ denote the map that is defined by $\alpha$ and let $\sigma^{\prime}$ denote the one defined $\psi^{*} \alpha$. A homotopy from $h$ to $h^{\prime}$ would give one from $\sigma$ and $\sigma^{\prime}$ were $\sigma=\sigma^{\prime}$ on $L$. However, such need not be the case. To address this concern, note first that $\sigma$ and $\sigma^{\prime}$ agree on the boundary of the unit ball, and also on the parts of $L$ that comprise boundaries of the balls $b_{0}, b_{1}$ and $b_{2}$. The maps $\sigma$ and $\sigma^{\prime}$ can only differ on the three arcs that connect $\partial b_{0}, \partial b_{1}$ and $\partial b_{2}$ to the origin. Of course, $\sigma$ and $\sigma^{\prime}$ agree on the endpoints of these arcs. In any event, the map $\sigma^{\prime}$ can be homotoped rel $\partial S$ so that the result, $\sigma^{\prime \prime}$, differs from $\sigma^{\prime}$ only in a small tubular neighborhood of each arc from $L$ and agrees with $\sigma$ on $L$. This is because the image space $S^{2}$ is simply connected. This map $\sigma^{\prime \prime}$ produces a corresponding map, $h^{\prime \prime}: S^{2} \times[0,1] \rightarrow S^{2}$. The issue here is whether $h$ and $h^{\prime \prime}$ are homotopic.

To see that such is the case, $\sigma^{\prime \prime}$ will be constructed in steps, and each step will results in a corresponding map from $S^{2} \times[0,1]$ that is homotopic to $h^{\prime}$ rel $S^{2} \times\{0,1\}$. The first step homotopes $\sigma^{\prime}$ to a map, $\sigma_{1}$, that agrees with $\sigma^{\prime}$ on the complement of a very small radius ball about the origin and also at the origin; but differs in being constant on a small neighborhood of the origin. This can be done so that $\sigma_{1}$ is as close as desired to $\sigma^{\prime}$ in the $C^{0}$ topology. The result gives a map, $h_{1}$, that is $C^{0}$ close to $h^{\prime}$ and so is homotopic to $h^{\prime}$ rel $\partial S$.

The second step changes $\sigma_{1}$ to $\sigma_{2}$. The map $\sigma_{2}$ can be made as close as desired to $\sigma_{1}$ in the $C^{0}$ topology. In particular, it agrees with $\sigma_{1}$ on the complement of the union of a small radius tubular neighborhood of each arc in $L$ and it agrees with $\sigma_{1}$ on each such arc. However, in a very small radius neighborhood of each such arc from $L$, the map $\sigma_{2}$ depends only on the affine coordinate along the central arc. The fact that $\sigma_{2}$ is $C^{0}$ close to $\sigma_{1}$ implies that the corresponding $h_{2}$ is homotopic to $h_{1}$.

The final step changes $\sigma_{2}$ to $\sigma^{\prime \prime}$. The map $\sigma^{\prime \prime}$ agrees with $\sigma_{2}$ on the complement of a very small tubular neighborhood of each arc in $L$. It is assumed in what follows that the radius of these neighborhoods is chosen so that in any such neighborhood, the map $\sigma_{2}$ depends only on the affine coordinate along the central arc. Inside such a tubular neighborhood, the map $\sigma^{\prime \prime}$ depends only on the affine coordinate along the central arc and on the radial coordinate on the transverse disks to the arc. Meanwhile, $\sigma^{\prime \prime}$ agrees with $\sigma$ along each $\operatorname{arc}$ in $L$. Now, there is no reason for $\sigma^{\prime \prime}$ to be $C^{0}$ close to $\sigma_{2}$ since there is no apriori reason for $\alpha$ and $\psi^{*} \alpha$ to be close along these arcs. However, where $\sigma^{\prime \prime}$ differs from $\sigma_{2}$, both maps factor through a two dimensional space. Here is why: Where $\sigma_{2} \neq \sigma^{\prime \prime}$, the map $\sigma_{2}$ depends only on the affine coordinate along each arc of $L$, and $\sigma^{\prime \prime}$ depends only on the latter coordinate and on the radial coordinate on the transverse disks. As a consequence, the corresponding maps $h_{2}$ and $h^{\prime \prime}$ can be taken so as to differ only where they both factor through a 2-dimensional space. This implies that the volume form on $S U(2)$ is pulled back as zero by both $h_{2}$ and $h^{\prime \prime}$ where these two maps differ. As a consequence, $n_{h_{2}, h^{\prime \prime}}=0$ and so $h_{2}$ and $h^{\prime \prime}$ are homotopic as desired.

Part 5: This part concern the existence of the diffeomorphism $\phi$. To begin, recall that Lemma 3.1 asserts that $\alpha$ and $\psi^{*} \alpha$ define 2-plane fields on $S$ that are homotopic rel $\partial S$. As both define overtwisted contact plane fields, a theorem of Eliashberg (Theorem 3.1.1 in [E]) asserts that these contact 2-plane fields are homotopic as contact 
fields on $S$ via an homotopy that restricts to the identity on $\partial S$. This understood, a theorem of Gray [Gr] asserts that there exists a diffeomorphism, this being $\phi$, that restricts as the identity on a neighborhood of the 4 points in $\partial B_{1}$ where $\omega=0$, and pulls back the kernel of $\psi^{*} \alpha$ as the kernel of $\alpha$ where these 1-forms are non-zero. The fact that $\phi^{*} \psi^{*} \operatorname{kernel}(\alpha)=\operatorname{kernel}(\alpha)$ and $\phi^{*} \psi^{*} \alpha=\alpha$ near the zeros of $\omega$ imply that

$$
\phi^{*} \psi^{*} \alpha=g \alpha
$$

where $g$ is a strictly positive function on $\partial B^{1}$.

Part 6: Fix some very small but positive number $\varepsilon_{2}$ and use the exponential map from the metric to trivialize a tubular neighborhood of $\partial B_{1}$ as $\left(-\varepsilon_{2}, \varepsilon_{2}\right) \times \partial B_{1}$ so that $\partial B_{1}$ identified with $\{0\} \times \partial B_{1}$. This trivialization can and should be chosen with the following property: Near each zero of $\omega$ in $\partial B_{1}$, the fibers of the projection to $\partial B_{1}$ appear in the coordinates $(r, \lambda, \varphi, z)$ that are used in (3.9) as the loci where $(\lambda, \varphi, z)$ is constant. In what follows, $s$ denotes the coordinate on $\left(-\varepsilon_{2}, \varepsilon_{2}\right)$. Near each zero of $\omega$ where the projection sends $(r, \lambda, \varphi, z)$ to $(\lambda, \varphi, z)$, the coordinate $s$ is taken to be $r-\varepsilon$.

Extend $\phi$ to this tubular neighborhood as the identity on the $\left(-\varepsilon_{2}, \varepsilon_{2}\right)$ factor. Doing so produces two symplectic forms on the $\left(-\varepsilon_{2}, 0\right)$ portion of the tubular neighborhood; the first being $\omega$, and the second $\phi^{*} \psi^{*} \omega$. Note that they agree on the portion of the tubular neighborhood that lies over any small radius ball in $\partial B_{1}$ about a zero of $\omega$.

Let $s$ now denote the coordinate on $\left(-\varepsilon_{2}, \varepsilon_{2}\right)$ and write

$$
\omega=d(\alpha+s b)
$$

where $b$ is a smooth $s$-valued 1 -form on $\partial B_{1}$. In this regard,

$$
\left.b\right|_{s=0} \wedge d \alpha \geq 0
$$

with equality only on the four zeros of $\omega$ in $\partial B_{1}$. Note that over a small radius ball in $\partial B_{1}$ about a zero of $\omega$, one can assume without loss of generality that $\alpha+s b=\alpha_{+}$, this the form that is depicted in (3.9).

Meanwhile, $\phi^{*} \psi^{*} \omega$ can be written as

$$
\phi^{*} \psi^{*} \omega=d\left(g \cdot \alpha+s b^{\prime}\right)
$$

where $b^{\prime}$ is another smooth, $s$-valued 1 -form on $\partial B_{1}$. In this case,

$$
\left.b^{\prime}\right|_{s=0} \wedge(d g \wedge \alpha+g d \alpha) \geq 0
$$

with equality only on the zeros of $\omega$ in $\partial B_{1}$. As in the case with $\omega$, there is no generality lost by taking $g \cdot \alpha+s b^{\prime}=\alpha_{+}$over a small radius ball about a zero of $\omega$.

Now, let $\beta$ denote a non-decreasing, smooth function on $\left[-\varepsilon_{2}, \varepsilon_{2}\right]$ that is 0 near $-\varepsilon_{2}$ and 1 on $\left[0, \varepsilon_{2}\right]$. In particular, given some positive $\varepsilon_{3} \ll \varepsilon_{2}$, choose $\beta$ so that $\beta^{\prime}=1 / \varepsilon_{2}$ where $-\varepsilon_{2}+\varepsilon_{3} \leq s \leq-\varepsilon_{3}$ and $\beta^{\prime}<2 / \varepsilon_{2}$ everywhere. Let $\kappa$ denote a positive number that is less than 1 and consider

$$
\begin{aligned}
\mu= & d\left[\beta(\alpha+s b)+\kappa(1-\beta)\left(g \alpha+s b^{\prime}\right)\right] \\
= & d s \wedge\left[\beta b+\kappa(1-\beta) b^{\prime}+\beta^{\prime}\left((1-\kappa g) \alpha+s b-\kappa s b^{\prime}\right)\right] \\
& +[\beta+\kappa g(1-\beta)] d \alpha+\kappa(1-\beta) d g \wedge \alpha+\cdots,
\end{aligned}
$$


where the unwritten terms are $\mathcal{O}\left(\varepsilon_{2}\right)$ in size. The important point here is that the form depicted in (3.30) is symplectic except at the zeros of $\omega$ if $\kappa<\sup (g), \varepsilon_{2}$ is very small, and then $\varepsilon_{3}$ very much smaller than $\varepsilon_{2}$. To see why this is, consider first the story near a zero of $\omega$. Near such a point, $g=1$, the coordinate $s=r-\varepsilon$, and

$$
\mu=(\beta+\kappa(1-\beta)) \omega+d r \wedge \beta^{\prime}(1-\kappa) \alpha_{+} .
$$

Given (3.7), this finds

$$
\begin{aligned}
\mu= & d r \wedge\left[(\beta+\kappa(1-\beta))+\frac{1}{3} r \beta^{\prime}(1-\kappa)\right] r^{2} \sin (\lambda) d \lambda \\
& -d r \wedge\left[(\beta+\kappa(1-\beta))+r \beta^{\prime}(1-\kappa)\right] 2 z \cos (\lambda) d z \\
& -d r \wedge\left[(\beta+\kappa(1-\beta))+r \beta^{\prime}(1-\kappa)\right] z \sin ^{2}(\lambda) d \varphi \\
& +2 z r \sin (\lambda) d \lambda d z+r^{2} \sin ^{2}(\lambda) d \varphi d z-2 z r^{2} \sin (\lambda) \cos (\lambda) d \lambda d \varphi .
\end{aligned}
$$

When $\kappa<1$, the square of this form is positive except on the line segments where both $z$ and $\sin (\lambda)$ are 0 . This follows from the fact that $\beta^{\prime} \geq 0$. In particular, (3.32) defines a near symplectic form when $\kappa<1$.

The next point to make is that $\mu \wedge \mu$ is strictly positive away from the zero locus of $\omega$. To see why, remark that if $\kappa<\sup (g)$ and $\varepsilon_{2}$ is very small, then $\mu \wedge \mu$ is dominated by the following part of $\mu$

$$
\beta(1-\kappa g) d s \wedge \alpha+[\beta+\kappa g(1-\beta)] d \alpha+\kappa(1-\beta) d g \wedge \alpha
$$

except where $\beta^{\prime}$ is on the order of unity or smaller. If $\varepsilon_{3}$ is much smaller than $\varepsilon_{2}$, then the latter region has $\beta$ very close to 1 and $(1-\beta) \sim \varepsilon_{3} / \varepsilon_{2}$ or $(1-\beta)$ very close to 1 and $\beta \sim \varepsilon_{3} / \varepsilon_{2}$. In the former case, terms with $(1-\beta)$ are negligible, and

$$
\mu \sim d s \wedge\left[\beta b+\beta^{\prime}\left((1-\kappa g) \alpha+s b-\kappa s b^{\prime}\right)\right]+\beta d \alpha .
$$

This understood, the fact that $\mu$ is near symplectic here follows from (3.27). In the case where $\beta$ is very small,

$$
\mu \sim d s \wedge\left[\kappa(1-\beta) b^{\prime}+\beta^{\prime}\left((1-\kappa g) \alpha+s b-\kappa s b^{\prime}\right)\right]+\kappa(1-\beta)(g d \alpha+d g \wedge \alpha) .
$$

Here, the fact that $\mu$ is near symplectic follows from (3.29).

Note that $\mu=\omega$ where $s \geq 0$ and $\mu=\phi^{*} \psi^{*} \omega$ where $s$ is very near $-\varepsilon_{2}$.

Acknowledgement. The author thanks Rosa Sena-Dias for discussions about the theorem of Luttinger and Simpson.

\section{References}

[ADK] D. Auroux, S.K. Donaldson and L. Katzarkov, Singular Lefschetz pencils, Geom. Topol. 9 (2005) 1043-1114.

[DK] S.K. Donaldson and P.B. Kronheimer, The geometry of 4-manifolds, Oxford University Press, 1990.

[E] Y. Eliashberg, The classification of overtwisted contact structures on 3-manifolds, Invent. Math. 98 (1989) 623-637.

[Gr] J. Gray, Some global properties of contact structures, Ann. Math. 69 (1959) 421-450.

[G] M. Gromov, Pseudo-holomorphic curves in symplectic manifolds, Invent. Math. 82 (1985) 307-347.

[Ha] A. Hatcher, A proof of a Smale conjecture, Diff $\left(S^{3}\right) \simeq S O(4)$, Ann. of Math. 117 (1983) 553-607. 
[Ho] K. Honda, An openness theorem for harmonic 2-forms on 4-manifolds, Illinois J. Math. 44 (2000) 479-495.

[K] P.B. Kronheimer, Minimal genus in $S^{1} \times M^{3}$, Invent. Math. 135 (1999) 45-61.

[KG] D. Gay and R. Kirby, Constructing symplectic forms on 4-manifolds which vanish on circles, Geom. Topol. 8 (2004) 743-777.

[M] J.K. Moser, On the volume of manifolds, Transactions of the A.M.S. 120 (1965) 280-296.

[P] T. Perutz, Zero sets of near symplectic forms, math.SG/0601320.

[S] R.H. Scott, Closed, self-dual two-forms on four-dimensional handlebodies, Ph.D. Thesis, Harvard Univerity 2003.

[T1] C.H. Taubes, The geometry of the Seiberg-Witten invariants, in the Proceedings of the International Congress of Mathematicians, Berlin 1998, Vol II, Documenta Mathematica Extra Volume ICM 1998, 493-504.

[T2] The Seiberg-Witten invariants and symplectic forms, Math. Res. Lett. 1 (1994) 809-822.

[T3] More constraints on symplectic forms from the Seiberg-Witten invariants, Math. Res. Lett. 2 (1995) 9-13.

Department of Mathematics, Harvard University, Cambridge, MA 02138

E-mail address: chtaubes@math.harvard.edu 